\title{
97. Note on Dirichlet Series, $X$. Remark on S. Mandelbrojt's Theorem
}

\author{
By Chuji Tanaka
}

Mathematical Institute, Waseda University, Tokyo

(Comm. by Z. SuetunA, M.J.A., Oct. 12, 1953)

(1) Introduction. Let us put

(1. 1) $F(s)=\sum_{n=1}^{\infty} a_{22} \exp \left(-\lambda_{n} s\right) \quad\left(s=\sigma+i t, 0 \leqq \lambda_{1}<\lambda_{2}<\cdots<\lambda_{n} \rightarrow+\infty\right)$.

Let $F(s)$ be uniformly convergent in the whole plane. Then $F(s)$ defines the integral function, and for any given $\sigma, \operatorname{Sup}_{-\infty<t<+\infty}|F(\sigma+i t)|$ has the finite value $M(\sigma)$. After J. $\operatorname{Ritt}^{1)}$ (pp. 18-19), we can define the order and type of $F(s)$ as follows:

Definition I. The order $\sigma$ of (1.1) is defined by

$$
\rho=\varlimsup_{\sigma \rightarrow-\infty} 1 /(-\sigma) . \log ^{+} \log ^{+} M(\sigma),
$$

where $\log ^{+} x=\operatorname{Max}(0, \log x)$. If $0<\rho<+\infty$, then the type $k$ of (1.1) is defined by

$$
k=\varlimsup_{\sigma \rightarrow-\infty} 1 / \exp ((-\sigma) \rho) . \log ^{+} M(\sigma) .
$$

Definition II. Let $D(r ; C)$ be the curved strip which is generated by circles with radii $r$, and having its centres on the analytic curve $C$, which extends to $\Re(s)=-\infty$. Then the order $\rho(D)$ in $D$ is defined by

$$
\rho(D)=\lim 1 /(-\sigma) . \log ^{+} \log ^{+} M(\sigma ; D),
$$

where $M(\sigma ; D)=\operatorname{Max}|F(s)|$. If $0<\rho(D)<+\infty$, then the type $k(D)$ in $D$ is defined by

$$
k(D)=\varlimsup_{\sigma \rightarrow-\infty} 1 / \exp ((-\sigma) \rho(D)) . \log ^{+} M(\sigma ; D) .
$$

S. Mandelbrojt has proved the following.

Theorem (S. Mandelbrojt ${ }^{1)}$ p. 19). Let (1. 1) with $\lim _{n \rightarrow+\infty}\left(\lambda_{n+1}-\lambda_{n}\right)=$ $h>0, \varlimsup_{n \rightarrow+\infty} n / \lambda_{n}=\delta(\leqq 1 / h)$ be simply (necessarily absolutely) convergent in the whole plane. Then, in any strip: $|\Im(s)-t| \leqq \pi(\delta+\varepsilon)(t$ : arbitrary but fixed, $\varepsilon$ : any given positive constant), (1.1) has the same order as in the whole plane.

In this note, we shall generalize it as follows:

Theorem. Let (1. 1) with $\lim _{n \rightarrow+\infty}\left(\lambda_{n+1}-\lambda_{n}\right)=h>0, \varlimsup_{n \rightarrow+\infty} n / \lambda_{n}=\delta(\leqq$ $1 / h)$ be simply (necessarily absolutely) convergent in the whole plane. Then, in any curved strip $D(\pi(\delta+\varepsilon) ; C)(\varepsilon$ : any given positive constant), (1.1) has the same order as in the whole plane.

If furthermore $\delta=0$, then in $D(\varepsilon ; C),(1.1)$ has the same order and type as in the whole plane. 
Remark. G. Pólya ${ }^{2)}$ (p. 627) has proved the second part of this theorem in the case of Taylor series by the very complicated method.

(2) Lemma. We shall establish next lemma, which is a generalization of J. J. Gergen-S. Mandelbrojt's theorem ${ }^{133)}$. ( 1) pp. 13-14, 3) pp. 4-6).

Lemma. Under the same conditions as in the theorem, we have

$$
\operatorname{Sup}_{\Re(s)=\Re\left(s_{0}\right)}\left|F^{\prime}(s)\right| \leqq A \cdot \operatorname{Max}_{\mid u-s_{1}=\pi(\sigma+\varepsilon)}|F(u)|
$$

where

(i) $s_{0}, s_{1}$ : two arbitrary points, but $\mathfrak{R}\left(s_{1}\right)=\mathfrak{R}\left(s_{0}\right)$

$-\left(3 \delta \log \left(e^{8} / h \delta\right)+2 \varepsilon\right)$,

(ii) $A$ : constant depending upon only $\varepsilon, \delta$ and $\left\{\lambda_{n}\right\}$.

Proof. By $\varlimsup_{n \rightarrow+\infty} n / \lambda_{n}=\delta<+\infty, \sum_{n=1}^{\infty} 1 / \lambda_{n}^{2}$ converges, so that, putting

$$
\varphi_{n}(z)=\prod_{\substack{\nu=1 \\ \nu \neq n}}^{\infty}\left(1-z^{2} / \lambda_{\nu}^{2}\right)
$$

(2.1) is an integral function. Hence, by F. Carlson-A. Ostrowski's theorem ${ }^{4)}$ (p. 267), for any given $\varepsilon(>0)$, we have

$$
\left|\varphi_{n z}(z)\right|<\exp (\pi(\delta+\varepsilon)|z|) \text { for }|z|>R(\varepsilon) .
$$

Setting $\varphi_{n}(z)=\sum_{\nu=0}^{\infty} c_{\nu}^{(n)} / \nu ! . z^{\nu}$, by Cauchy's theorem and (2.2), we get easily

$$
\left|c_{\nu}^{(r)} / \nu !\right|<1 / r^{\nu} . \exp (\pi(\delta+\varepsilon) r) \quad r=|z| .
$$

Since the right-hand side takes its minimum at $r=\nu / \pi(\delta+\varepsilon)$, for sufficiently large $\nu$, we have

$$
\left|c_{\nu}^{(n)}\right|<\{\pi(\delta+2 \varepsilon)\}^{\nu} .
$$

Hence, there exists a constant $K_{1}(\varepsilon)$ such that

$$
\left|c_{\nu}^{(n)}\right|<K_{1}(\varepsilon) .\{\pi(\delta+2 \varepsilon)\}^{\nu} \quad(\nu=1,2, \ldots) .
$$

Putting $\Phi_{n}(z)=\sum_{\nu=0}^{\infty} c_{\nu}^{(n)} / z^{\nu+1}$, by (2.3), $\Phi_{n}(z)$ is convergent for $|z|>\pi \delta$. On account of H. Cramer-A. Ostrowski's theorem ${ }^{4)}$ (pp. 49-52), we have

so that, by (2.3)

$$
\begin{aligned}
a_{n} \varphi_{n}\left(\lambda_{n}\right) \exp \left(-\lambda_{n} s\right) & =\sum_{\nu=1}^{\infty} a_{\nu} \phi_{n 2}\left(\lambda_{\nu}\right) \exp \left(-\lambda_{\nu} s\right) \\
& =1 / 2 \pi i \cdot \oint_{|u|=\pi(\delta+3 \varepsilon)} F(s-u) \Phi_{n 2}(u) d u,
\end{aligned}
$$

$$
\begin{gathered}
\left|a_{n} \phi_{n}\left(\lambda_{n}\right) \cdot \exp \left(-\lambda_{n} s\right)\right| \\
\leqq \operatorname{Max}_{|s-u|=\pi(\delta+3 \varepsilon)}|F(u)| \cdot 1 / 2 \pi \cdot \oint_{|u|=\pi(\delta+3 \varepsilon)}\left\{\sum_{\nu=0}^{\infty}\left|c_{\nu}^{(n)}\right| u^{\nu+1} \mid\right\}|d u| \\
<\operatorname{Max}_{|s-u|=\pi(\delta+8 \varepsilon)}|F(u)| \cdot K_{1}(\varepsilon) \cdot \sum_{\nu=0}^{\infty}\{(\delta+2 \varepsilon) /(\delta+3 \varepsilon)\}^{\nu} .
\end{gathered}
$$

Therefore, replacing $\varepsilon$ by $\varepsilon / 3$, we can put 


$$
\left|a_{n 2} \varphi_{n}\left(\lambda_{n}\right) \exp \left(-\lambda_{n} s\right)\right| \leqq C \operatorname{Max}_{|s-u|=\pi(\delta+\varepsilon)}|F(u)|,
$$

where $C$ : a constant depending upon only $\varepsilon$ and $\delta$.

On the other hand, by F. Carlson-A. Ostrowski's theorem ${ }^{4)}$ (p. 267) for any given $\varepsilon(>0)$, and sufficiently large $\lambda_{2}$, we get

$$
\left|1 / \phi_{n z}\left(\lambda_{12}\right)\right|<\exp \left\{\left(3 \delta \log \left(e^{6} / h \delta\right)+\varepsilon\right) \lambda_{n}\right\} .
$$

Accordingly, there exists a constant $K_{2}(\varepsilon)$ such that

$$
\left|1 / \phi_{n}\left(\lambda_{n}\right)\right|<K_{2}(\varepsilon) \exp \left\{\left(3 \delta \log _{(n=1,2, \ldots)}\left(e^{\theta} / h \delta\right)+\varepsilon\right) \lambda_{n}\right\} .
$$

By (2.4), in which we put $s=s_{1}\left(\Re\left(s_{1}\right)=\Re\left(s_{0}\right)-\left(3 \delta \log \left(e^{\natural} / h \delta\right)+2 \epsilon\right)\right)$, we obtain

so that

$$
\begin{gathered}
\left|a_{n 2}\right| \exp \left(-\lambda_{12} \Re\left(s_{0}\right)\right) \cdot \exp \left\{\lambda_{n}\left(3 \delta \log \left(\epsilon^{\theta} / h \delta\right)+2 \varepsilon\right)\right\} \\
\leqq\left|1 / \phi_{n}\left(\lambda_{n 2}\right)\right| \cdot C . \operatorname{Max}_{\mid u-s_{1}=\pi(\delta+\varepsilon)}|F(u)|,
\end{gathered}
$$

Hence,

$$
\left|a_{n 2}\right| \exp \left(-\lambda_{n} \Re\left(s_{0}\right)\right) \leqq \exp \left(-\lambda_{22} \varepsilon\right) \cdot K_{2} \cdot C \cdot \operatorname{Max}_{|u-s|=\pi(\delta+\varepsilon)}|F(u)| .
$$

$$
\begin{gathered}
\operatorname{Sup}_{\Re(s)=\Re\left(s_{0}\right)}|F(s)| \\
\leqq \sum_{n=1}^{\infty}\left|a_{n}\right| \exp \left(-\lambda_{n} \Re\left(s_{0}\right)\right) \\
\leqq\left\{\sum_{n=1}^{\infty} \exp \left(-\lambda_{n} \varepsilon\right)\right\} . K_{2} \cdot C \cdot \operatorname{Max}_{\left|u-s_{1}\right|=\pi(\delta+\varepsilon)}|F(u)| .
\end{gathered}
$$

By G. Valiron's theorem ${ }^{4)}$ (p. 4) and $\lim \log n / \lambda_{2}=0$, the simple convergence-abscissa $\sigma_{s}$ of $\sum_{n=1}^{\infty} \exp \left(-\lambda_{2} s\right)$ is given by

$$
\sigma_{s}=\varlimsup_{n \rightarrow \infty} 1 / \log \lambda_{22} . \log 1=0,
$$

so that $\sum_{n=1}^{\infty} \exp \left(-\lambda_{n} \varepsilon\right)<+\infty$. Thus, by (2.6) we get

$$
\operatorname{Sup}_{\mathfrak{R}(s)=\Re\left(s_{0}\right)}|F(s)| \leqq A \operatorname{Max}_{\left|w-s_{1}\right|=\pi(\delta+\varepsilon)}|F(u)| \text {. }
$$

(3) Proof of theorem.

$$
\text { q.e.d. }
$$

(I) Let (1.1) be of order $\rho$. Then, by definition, there exists at least one sequence $\left\{\sigma_{m}\right\}$ such that

(i) $\lim _{m \rightarrow+\infty} \sigma_{m}=-\infty$

(ii) $\rho=\lim _{m \rightarrow+\infty} 1 /\left(-\sigma_{m}\right) \cdot \log ^{+} \log ^{+} M\left(\sigma_{m}\right)$.

Let us define two points $s_{0}, s_{1}$ on $C$ such that

$$
\begin{aligned}
& \text { (i) } s_{0}=\sigma_{m}+i t_{m}, \\
& \text { (ii) } \mathfrak{R}\left(s_{1}\right)=\mathfrak{R}\left(s_{0}\right)-(r(\delta)+\varepsilon), r(\delta)=3 \delta \log \left(e^{\natural} / h \delta\right) .
\end{aligned}
$$

By lemma, in which we replace $\varepsilon$ by $\varepsilon / 2$, we get

Therefore, putting $\Re\left(s_{1}^{\prime}\right)=\sigma_{m}^{\prime}$, we get

$$
M\left(\sigma_{m}\right) \leqq A . \operatorname{Max}_{\left|u-s_{1}\right|=\pi(\delta+\varepsilon / 2)}|F(u)|=A \cdot \underset{\left|s_{1}^{\prime}-s_{1}\right|=\pi(\delta+\varepsilon / 2)}{\left|F\left(s^{\prime}\right)\right|}
$$

$$
M\left(\sigma_{m}\right) \leqq A\left|F\left(s_{1}^{\prime}\right)\right| \leqq A M\left(\sigma_{m}^{\prime} ; D\right),
$$


where $M\left(\sigma_{m}^{\prime} ; D\right)=\operatorname{Max}_{\Re(s)=\sigma^{\prime}}|F(s)|$, so that, by (3.1)

$$
\begin{aligned}
\rho & =\lim _{m \rightarrow+\infty} 1 /\left(-\sigma_{m}\right) . \log ^{+} \log ^{+} M\left(\sigma_{m}\right) \\
& \leqq \varlimsup_{m \rightarrow+\infty} 1 /\left(-\sigma_{m}^{\prime}\right) . \log ^{+} \log ^{+} M\left(\sigma_{m}^{\prime} ; D\right) . \varlimsup_{m \rightarrow+\infty}\left(\sigma_{m}^{\prime} / \sigma_{m}\right) .
\end{aligned}
$$

Since $\left|\sigma_{m}-\sigma_{m}^{\prime}\right| \leqq r(\delta)+\varepsilon+\pi(\delta+\varepsilon / 2)$, we have evidently

Hence, by (3.2),

$$
\lim _{m \rightarrow+\infty} \sigma_{m}^{\prime} / \sigma_{m}=1 \text {. }
$$

$$
\rho \leqq \overline{\lim }_{m \rightarrow+\infty} 1 /\left(-\sigma_{m}^{\prime}\right) . \log ^{+} \log ^{+} M\left(\sigma_{m}^{\prime} ; D\right) \leqq \varlimsup_{\sigma \rightarrow-\infty} 1 /(-\sigma) . \log ^{+} \log ^{+} M(\sigma ; D) \text {. }
$$

Since the opposite inequality is evident, the equality holds, which proves the first part of theorem.

(II) Let (1.1) with $\delta=0$ be of order $\rho(0<\rho<+\infty)$, and of type $k$. Then, by definition, there exists at least one sequence $\left\{\sigma_{m}\right\}$ such that

$$
\begin{aligned}
& \text { (i) } \lim _{m \rightarrow+\infty} \sigma_{m}=-\infty \\
& \text { (ii) } k=\lim _{m \rightarrow+\infty} 1 / \exp \left(\left(-\sigma_{m}\right) \rho\right) \cdot \log ^{+} M\left(\sigma_{m}\right) .
\end{aligned}
$$

We define two points $s_{0}, s_{1}$ on $C$ such that

(i) $s_{0}=\sigma_{m}+i t_{m}$

(ii) $\Re\left(s_{1}\right)=\Re\left(s_{0}\right)-\varepsilon^{\prime} / \pi . \quad\left(0<\varepsilon^{\prime}<\varepsilon\right)$

Applying lemma, in which we replace $\varepsilon$ by $\varepsilon^{\prime} / 2 \pi$, we get

$$
M\left(\sigma_{m}\right) \leqq A \operatorname{Max}_{\left|u-s_{1}\right|=\varepsilon^{\prime} / 2}|F(u)|=A \underset{\left|s_{1}^{\prime}-s_{1}\right|=\varepsilon^{\prime} / 2}{\left|F\left(s_{1}^{\prime}\right)\right|,}
$$

so that, putting $\sigma_{m}^{\prime}=\Re\left(s_{1}^{\prime}\right), \quad M\left(\sigma_{m}\right) \leqq A . M\left(\sigma_{m}^{\prime} ; D\right)$.

Hence, by (3.3)

$$
\begin{aligned}
& k=\lim _{m \rightarrow+\infty} 1 / \exp \left(\left(-\sigma_{m}\right) \rho\right) \cdot \log ^{+} M\left(\sigma_{m}\right) \\
& \leqq \varlimsup_{m \rightarrow+\infty} 1 / \exp \left(\left(-\sigma_{m}^{\prime}\right) \rho\right) \cdot \log ^{+} M\left(\sigma_{m}^{\prime} ; D\right) \varlimsup_{m \rightarrow+\infty} \exp \left(\left(\sigma_{m}-\sigma_{m}^{\prime}\right) \rho\right) \\
& \leqq \varlimsup_{\sigma \rightarrow-\infty} 1 / \exp ((-\sigma) \rho) \cdot \log ^{+} M(\sigma ; D) \cdot \exp \left(\varepsilon^{\prime}(1 / \pi+1 / 2) \rho\right) \\
& =k(D) \cdot \exp \left(\varepsilon^{\prime}(1 / \pi+1 / 2) \rho\right) .
\end{aligned}
$$

Letting $\varepsilon^{\prime} \rightarrow 0$,

$$
k \leqq k(D) \text {. }
$$

Since the opposite inequality is evident, the equality holds, which proves the second part of theorem.

\section{References}

1) S. Mandelbrojt: "Séries lacunaires", Actualités scientifiques et industrielles, Paris, 305 (1936).

2) G. Pólya: ,Untersuchungen ùber Lücken und Singularitäten von Potenzreihen ", Math. Zeit., 29 (1929).

3) J. J. Gergen-S. Mandelbrojt: "On entire functions defined by a Dirichlet series", Amer. Jour. Math., 53 (1931).

4) V. Bernstein: "Leçons sur les progrès récents de la théorie des séries de Dirichlet", Collection Borel (1933). 